\title{
C-Reactive Protein Based on Injury Level and Physical Activity Level of Chronic Spinal Cord Injury Patient
}

\author{
Pricilya Morien Sangkoy ${ }^{\mathrm{a}}$, Hening Laswati Putra ${ }^{\mathrm{b} *}$, Nuniek Nugraheni $^{\mathrm{c}}$ \\ a,b,cDepartment of Physical Medicine and Rehabilitation, Faculty of Medicine Universitas Airlangga 60132, \\ Dr. Soetomo General Academic Hospital, Surabaya, Indonesia \\ *Corresponding Author: 1putra04@yahoo.com
}

\begin{abstract}
Background: Developing cardiovascular diseases such as arrhythmias, heart failure, and myocardial infarction are approximately 4 times greater in individuals with a spinal cord injury (SCI) than their age-matched peers without SCI. Spinal cord injury patients tend to have increased fat mass, urinary tract infections (UTI), pressure ulcers, and loss of function of major muscle groups which result in decreased levels of physical activity. All of these conditions can lead to increased levels of C-reactive protein (CRP) to the SCI patients. Elevated CRP levels are an independent risk factor contributing to the development of cardiovascular disease in this population. Levels of CRP on SCI patients are affected by several factors, they are the level of the lesion and the level of physical activity. Assessment of CRP levels can predict the risk of cardiovascular disease on SCI patients. The aim of this study was to obtain information about the differences in CRP level on SCI patients based on the level of the lesion and the level of physical activity. Early detection, prevention, and appropriate treatment will have a good impact on the health and quality of patient life with SCI.

Aim: Analysing the differences of C-reactive protein based on the level of injury and the level of physical activity of chronic spinal cord injury patients.

Methods: This research is a cross sectional study. The research subjects were 32 chronic SCI patients, consists of 18 men and 14 women, with injury duration of more than 1 year. Research subjects were divided based on the level of the lesion, that are lesion of the C3-T6 and T7-below. Physical activity levels were divided into active and less active group using International Physical Activity Questionnaire (IPAQ). Measurement of CRP on the subjects of this study was hs-CRP using the enzyme-linked immunosorbent assay (ELISA). Data is presented in mean \pm standard deviation and analysis using SPSS v.24.0.

Results: There was no significant difference in hs-CRP based on the level of injury between groups of C3-T6 and T7below (p 0.884). Patients of SCI of C3-T6 level with active physical activity levels had significantly lower hs-CRP values

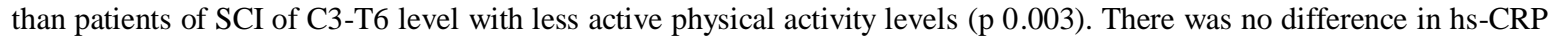
on the patients with SCI level T7-below between active and less active levels of physical activity (p 0.165).

Conclusion: The levels of hs-CRP on the chronic SCI patients did not differ based on the level of injury for age, body mass index, duration of injury, level of physical activity, etiology of SCI, AIS, and history of catheter use which were homogeneous in both groups. Chronic SCI patients with active physical activity levels had better hs-CRP than those who were less active in the SCI group of C3-T6.
\end{abstract}

Keywords: CRP; injury level; physical activity level; chronic spinal cord injury 


\section{Introduction}

Developing cardiovascular diseases such as arrhythmias, heart failure, and myocardial infarction are approximately 4 times greater in individuals with a spinal cord injury (SCI) than their age-matched peers without SCI (El-Kotob et al., 2018). This cardiovascular disorder is important because it causes high morbidity and mortality to the SCI patients (Calvo-Infante et al., 2018). The incidence of spinal cord injury in the world is recorded at $0,019 \%$ to $0,088 \%$ per year from data of thirty-five to fifty-three million people in the world (Widhiyanto et al., 2018). Meanwhile, the prevalence of symptomatic cardiovascular disease on SCI patients is 30-50 percent compared to 5-10 percent on the general population (Myers et al., 2007). The prevalence rate of asymptomatic cardiovascular disease is estimated to be around 60-70 percent on the SCI patients (Bauman et al., 1994). The death prevalence because of cardiovascular complications reaches up to 30 percent of deaths on the SCI (Calvo-Infante et al., 2018).

Systemic inflammation is associated with an increased risk of atherosclerosis and coronary heart disease. Systemic inflammation can be assessed by increasing levels of C-Reactive Protein (CRP). The increasing of CRP levels can predict future coronary events and other manifestations of cardiovascular disease in healthy individuals. The American Heart Association (AHA) has established clinical guidelines for CRP, namely low risk $<1 \mathrm{mg} / \mathrm{L}$, moderate risk 1-3 mg/L, high risk $>3 \mathrm{mg} / \mathrm{L}$ (Morse et al., 2008; Gibson et al., 2008).

Levels of CRP can predict cardiovascular disease risk on the SCI patients. CRP levels on the SCI patients are affected by several factors, namely the level of the lesion and the level of physical activity. CRP levels on the patients tetraplegia are higher than those in paraplegia. The high levels of CRP on the tetraplegia patients is due to their lower physical activity (Gibson et al., 2008). Based on physical activity, levels of CRP on the SCI patients who use wheelchairs are higher than those who walk with assistive devices. Wheelchair users tend to have lower levels of physical activity than those who walk with assistive devices (Morse et al., 2008). Decreased physical activity leads to an increase in adipose tissue. Adipose tissue has been shown to synthesize cytokines (IL-6, TNF- $\alpha$ ) which are involved in production of CRP (Vashist et al., 2015).

Previous studies compared the levels of CRP of SCI patients based on paraplegia and tetraplegia, but did not correlate it with levels of physical activity (Gibson et al., 2008). Another study tried to compare CRP levels with the level of physical activity of SCI patients, but the physical activity assessed was the type of mobilization of the patient, such as using a wheelchair or cane (Morse et al., 2008). Physical activity is not only determined by the type of mobilization but also other daily activities. In this study, the level of injury was differentiated based on the level of injury of C3-T6 and T7-below according to the cardiac autonomic level. Assessment of the level of physical activity in this study includes physical activity while sitting relaxed, physical activity in relation to mobilization, work, home/family care, sports and recreation.

Based on the description above, the aim of this study was to obtain the description of the differences of CRP on the chronic SCI patients based on the level of the lesion and the level of physical activity. Assessment of CRP levels can predict the risk of cardiovascular disease on the SCI patients. The inflammatory biomarker CRP can help stratify cardiovascular disease risk in chronic SCI patients and identify groups of patients who may benefit from physical activity. Early detection, prevention and appropriate treatment will have a good impact on the health and quality of life of SCI patients.

\section{Methods and Materials}

This research is a cross sectional study of 32 chronic SCI patients, consists of 18 men and 14 women, at Physical Medicine and Rehabilitation Outpatients Clinic of Dr. Soetomo General Academic Hospital Surabaya. Our research was conducted from September 2020 to October 2021. The inclusion criteria were as follows: (1) Chronic SCI patients (at least 1 year after the incident), (2) Injury level at C3 and below, (3) eighteen years old up to sixty-four year old, (4) SCI caused by traumatic or non-traumatic causes, regardless of gender, ethnicity, socioeconomic status, (5) good communication and cognitive, (6) Provide valid and 
signed informed consent prior to any study protocol. Exclusion criteria were as follows: (1) Patients with diabetes, heart problems, lung disorders, decubitus ulcers, (2) Patients with infection/fever $\left(>37,5^{\circ}\right)$ in the last 1 week.

The subjects were divided into two groups based on the level of the lesion, group C3-T6 and group T7below. The research subjects were SCI patients with ASIA impairment scale (AIS) A-D classification. Physical activity is defined as any body movement produced by skeletal muscles that results in energy expenditure (Caspersen et al., 1985). Physical activity was assessed using the IPAQ questionnaire, an independent standard measuring tool for the level of habitual physical activity consisting of 4 sets of questionnaires. The level of physical activity in this study was divided into two groups, the active group if the IPAQ score $\geq 600 \mathrm{MET}$-minute/week and the less active group if the IPAQ score <600 MET-minute/week (Hastuti, 2013). C-reactive Protein (CRP) is a homopentameric acute-phase inflammatory protein (Sproston et al., 2018). Enzyme-linked immunosorbent assay (ELISA) method to evaluate hs-CRP in mg/L units.

Statistical analysis using SPSS v.24.0. To analyse the difference of CRP based on the level of the lesion and the level of physical activity, the $\mathrm{T}$ test difference test was used for data with normal distribution and Mann Whitney for data not normally distributed. Significant if $p<0.05$. This research has received ethical approval from the Ethics Commission of Dr. Soetomo General Academic Hospital Surabaya with number 0051/KEPK/IX/2020 dated September $7^{\text {th }}, 2020$.

\section{Results}

\subsection{Characteristics of the research subjects}

The characteristics of participants are shown in table 1, the sampling method is consecutive sampling. The total number of research subjects who met the inclusion criteria and were willing to participate in this study were 32 people, consisting of 16 patients with SCI level C3-T6 and 16 patients with SCI level T7-below.

Normality test using Kolmogorov-Smirnov Monte Carlo obtained data that were normally distributed for age, body mass index, duration of injury, IPAQ score, and etiology. AIS classification data for group C3-T6 were normally distributed. The AIS classification data for group T7-below and catheter use both group were not normally distributed. There were no significant differences for age, duration of injury, body mass index, etiology, AIS classification and IPAQ scores in the two groups. All members in each group did not smoke. The characteristics of the two groups were homogeneous ( $\mathrm{p}$-value $>0.05$ ).

The mean age of the subjects was 39.81 \pm 14.84 years in the C3-T6 group and $38.56 \pm 14.22$ years in the T7below group. The mean duration of injury was $1.96 \pm 1.30$ years in the C3-T6 group and $2 \pm 1.46$ years in the T7-below group. The active C3-T6 group had an IPAQ score of 818.18 \pm 262.45 MET-minute/week and the less active C3-T6 group was 208.75 \pm 103.98 MET-minute/week. The active group T7-below had an IPAQ score of $862.81 \pm 323.42 \mathrm{MET}-$ minute/week and the less active T7-below group had 217.5 \pm 121.27 METminute/week. The etiology of spinal cord injury in the C3-T6 group were vertebral fracture $(6.25 \%)$, canal stenosis (18.75\%), TB spondylitis (31.25\%), tumour (6.25\%), AVM (6.25\%), cancer (12.5\%), spondylolisthesis $(6.25 \%)$, transverse myelitis $(6.25 \%)$, and syringomyelia $(6.25 \%)$. The etiology of spinal cord injury in the T7-below group were vertebral fracture $(25 \%)$, canal stenosis $(18.75 \%)$, TB spondylitis $(31.25 \%)$, tumor $(0 \%)$, AVM $(0 \%)$, cancer $(6.25 \%)$, spondylolisthesis $(6.25 \%)$, transverse myelitis $(12.5 \%)$, and syringomyelia $(0 \%)$. 
Table 1. Baseline characteristics and homogeneity based on injury level

\begin{tabular}{|c|c|c|c|}
\hline Characteristics & Injury level C3-T6 $(n=16)$ & Injury level T7-below $(\mathrm{n}=16)$ & $\mathbf{p}$ \\
\hline $\begin{array}{l}\text { Age in years } \\
(\text { mean } \pm \text { SD) }\end{array}$ & $39.81 \pm 14.84$ & $38.56 \pm 14.22$ & 0,736 \\
\hline $\begin{array}{l}\text { Length of injury in years } \\
(\text { mean } \pm \text { SD })\end{array}$ & $1.96 \pm 1.30$ & $2 \pm 1.46$ & 0,491 \\
\hline $\begin{array}{l}\text { Body mass index } \\
\mathrm{kg} / \mathrm{m}^{2}(\text { mean } \pm \mathrm{SD})\end{array}$ & $22.85 \pm 3.55$ & $22.27 \pm 3.40$ & 0,979 \\
\hline $\mathbf{A}$ & $2(12.5)$ & $2(12.5)$ & \\
\hline B & $2(12.5)$ & $2(12.5)$ & 0,514 \\
\hline $\mathbf{C}$ & $5(31.25)$ & $3(18.75)$ & \\
\hline D & $7(43.75)$ & $9(56.25)$ & \\
\hline
\end{tabular}

IPAQ score

METs-min/week

$($ mean \pm SD)

$$
\text { Active } 818.18 \pm 262.45
$$

$862.81 \pm 323.42$

0,871

Less Active $208.75 \pm 103.98$

$217.5 \pm 121.27$

Catheter use \%

$$
\begin{array}{cl}
\text { Yes } & 4(25) \\
\text { No } & 12(75)
\end{array}
$$

$$
\begin{aligned}
& 6(37.5) \\
& 10(62.5)
\end{aligned}
$$$$
0,446
$$

Etiology \%

$$
\begin{array}{rll}
\text { Spondylitis TB } & 5(31.25) & 5(31.25) \\
\text { Tumor } & 1(6.25) & 0 \\
\text { Spondylolisthesis } & 1(6.25) & 1(6.25) \\
\text { Fracture } & 1(6.25) & 4(25) \\
\text { Central canal stenosis } & 3(18.75) & 3(18.75) \\
\text { Transverse Myelitis } & 1(6.25) & 2(12.5) \\
\text { Cancer } & 2(12.5) & 1(6.25) \\
\text { AVM } & 1(6.25) & 0 \\
\text { Syringomyelia } & 1(6.25) & 0
\end{array}
$$

Smoking \%

$$
\begin{aligned}
\text { Yes } & 0 \\
\text { No } & 16(100)
\end{aligned}
$$$$
0
$$

16(100) 


\subsection{C-reactive protein based on injury level}

Table 2 shows the differences in hs-CRP based on the level of injury between groups C3-T6 and T7below. The normality test of the data showed that the data was normally distributed, so it was used using parametric statistical tests with independent T-test. The results of the analysis showed that there was no difference in hs-CRP based on the level of injury between groups of C3-T6 and T7-below (p 0.884).

Table 2. Comparison of hs-CRP based on injury level

\begin{tabular}{lcc}
\hline Injury level & hs-CRP $($ mean \pm SD) & p \\
& mg/L & \\
\hline C3-T6 $(\mathbf{n}=\mathbf{1 6})$ & $5.41 \pm 5.98$ & 0.884 \\
T7-below $(\mathbf{n}=\mathbf{1 6})$ & $5.15 \pm 4.21$ & \\
\hline
\end{tabular}

*Note: significance of $\mathrm{p}<0.05$

3.3. C-reactive protein based on physical activity level

3.3.1 C-reactive protein based on physical activity level on SCI level C3-T6

Table 3 shows the differences in hs-CRP based on the level of physical activity in the C3-T6 group. The normality test of the data showed that the data was normally distributed, so it was used parametric statistical tests with independent T-test. The results of the analysis showed that there was a significant difference in hsCRP based on the level of physical activity in the C3-T6 group (p 0.003).

Table 3. Comparison of hs-CRP based on physical activity level on SCI level C3-T6

\begin{tabular}{lcc}
\hline Physical Activity Level & $\begin{array}{c}\text { hs-CRP }(\text { mean } \pm \text { SD) } \\
\text { mg/L }\end{array}$ & p \\
\hline Active $(\mathbf{n}=8)$ & $1.02 \pm 0.56$ & $0.003^{*}$ \\
Less Active $(\mathbf{n}=8)$ & $9.81 \pm 5.67$ &
\end{tabular}

*Note: The difference is significant if the value of $\mathrm{p}<0.05$. Active: Score IPAQ>600 MET-minutes/week. Less Active: Score of IPAQ<600 MET-minutes/week

\subsubsection{C-reactive protein based on physical activity level on SCI level T7-below}

Table 4 shows the-differences in hs-CRP based on the level of physical activity in the group of T7-below. The normality test of the data showed that the data was normally distributed, so it was used parametric statistical tests with independent T-test. The results of the analysis showed that there was no significant difference in hs-CRP based on the level of physical activity in the group of T7-below (p 0.165).

Table 4. Comparison of hs-CRP based on physical activity level on SCI level T7-below

\begin{tabular}{lcc} 
Physical Activity Level & $\begin{array}{c}\text { hs-CRP (mean } \pm \text { SD) } \\
\text { mg/L }\end{array}$ & p \\
\hline Active (n=8) & $3.66 \pm 2.98$ & 0.165 \\
Less Active (n=8) & $6.63 \pm 4.90$ &
\end{tabular}

*Note: The difference is significant if the value of $\mathrm{p}<0.05$. Active: Score IPAQ>600 MET-minutes/week. Less Active: Score of IPAQ<600 MET-minutes/week. 


\section{Discussion}

\subsection{Characteristics of research subjects}

Comparison of characteristics between the two groups in this study compared by age, duration of injury, body mass index, AIS classification, etiology, IPAQ score, catheter use and smoking history showed that the characteristics of the two groups were homogeneous (table 1). Other factors that affect CRP such as fever, diabetes, pressure ulcers, comorbidities such as cardiac and respiratory disorders have been excluded from the study subjects so that they will not affect the assessment of CRP.

\subsection{C-reactive protein based on injury level}

There was no difference in hs-CRP based on the level of injury between groups C3-T6 and T7-below (p 0.884). The results of this study are same as those of Wang et al (2007) who explained that there was no difference in CRP based on the level of injury between groups of C3-T6 and T7-below. Huang et al (2008) also explain the same thing, that there is no difference in CRP based on the level of injury between the paraplegia and tetraplegia groups.

The results of Gibson et al (2008) differ from the results of this study which showed that the hs-CRP in tetraplegia was significantly higher than in paraplegia. The research from Gibson et al (2008) found that patients with high body mass index, waist circumference, and fat mass percentage tend to have higher hsCRP. The consequence of decreased physical activity due to loss of muscle mass due to paralysis below the level of injury causes patients with chronic SCI to tend to have more adipose tissue (Wang et al., 2007). Tetraplegic patients tend to be less active than paraplegic patients who can still use their upper limbs for activities (Ruiz-Ramie et al., 2019). It explains the high hs-CRP in tetraplegic patients due to lower physical activity than paraplegic patients. Physical activity level between groups C3-T6 and group T7-below were not significantly different in this study, as shown in table 1 . This caused hs-CRP levels did not significantly differ in these two groups of lesions.

Adipose tissue has been shown to synthesize cytokines (IL-6, TNF- $\alpha$ ) which are involved in CRP production (Vashist et al., 2015). Measurement of adipose tissue can use waist circumference, body mass index, Bioelectrical Impedance (BIA) and Dual X-ray Absorptiometry (DXA). Measurements with body mass index are widely used in several studies to describe whole body fat, although measurements with the DXA method are better for detecting fat mass (Lee et al., 2005; Browning et al., 2011). In this study, the measurement of body mass index was used. Table 1 proves that the body mass index in the two groups of lesions was not significantly different. The same body mass index in both groups was the reason that the hsCRP analysis in this study did not differ between levels of C3-T6 and T7-below.

Spinal cord injury (SCI) patients tend to have recurrent infections (urinary tract infections, pneumonia, pressure ulcers) which can lead to elevated CRP levels to the SCI patients (Morse et al., 2008; Goldstein et al., 2017). In this study, SCI patients with pressure ulcers and pneumonia were excluded from this study. In this study, we used data on catheter use for the risk of urinary tract infection in study subjects because it is known that catheter use is correlated with chronic bacteriuria and pyuria, which causes increased CRP levels associated with catheter use (Goldstein et al., 2017). Table 1 shows that the use of catheters in the two groups of lesions was not significantly different. No one smoked in either group. These factors caused the hs-CRP in the two groups of lesions to not differ significantly.

There were similarities in the classification of AIS and the etiology of SCI in these two groups. Several studies on CRP in SCI patients showed that CRP values were not significantly differ based on AIS classification (complete/incomplete) (Gibson et al., 2008; Morse et al., 2008; Goldstein et al., 2017). This also causes hs-CRP in the two groups of lesions not significantly different. 
4.3. C-reactive protein based on physical activity level

4.3.1. C-reactive protein based on physical activity level on SCI level C3-T6

Table 3 shows that there are significant differences in hs-CRP based on the level of physical activity in the group of C3-T6 (p 0.003). The active group of SCI level C3-T6 had significantly lower hs-CRP values than the less active group of SCI level C3-T6. The results of this study are similar to those of Goldstein et al (2017) who found that SCI patients with active levels of physical activity had better CRP values than those who were less active. Goldstein et al (2017) assessed the level of physical activity based on the type of mobility. Research by Goldstein et al (2017) shows that the average CRP value increases along with a decrease in mobility. Values of CRP on the SCI patients who use motorized wheelchairs are higher than those who walk with assistive devices. CRP levels in those who walk with an assistive device are higher than those who walk independently. Compare to this study, activity level was assessed using the IPAQ score which assessed physical activity not only based on walking mobility but also activities at work, home, sports and hobbies.

Physical activity has a positive effect on health status and is associated with a reduced risk of heart disease. The relationship between physical activity and reduced risk of heart disease is mediated by the antiinflammatory effect of physical activity. Many studies have found an inverse relationship between physical activity and hs-CRP. Many studies in able-bodied people have found higher CRP levels to be associated with lower levels of physical activity. Several studies have shown that physical activity reduces CRP levels (Plaisance et al., 2006; Morse et al., 2008; Majka et al., 2009). Physical activity can reduce the number of proinflammatory cytokines (IL-1, IL6 and TNF $\alpha$ ) and reduce the production of CRP from the liver (Queiroz et al., 2020).

Cardiac sympathetic autonomic function is regulated at the T1-T6 level (Furlan et al., 2008). Injury at or above this level causes impaired supraspinal sympathetic control of the heart, resulting in increased sympathetic activity (West et al., 2012). Adrenergic dysfunction, poor diet and physical inactivity also have important roles in the increased risk of cardiovascular disease in SCI (Bravo et al., 2004, Warburton et al., 2007b). Several studies have shown that excessive sympathetic activity is associated with elevated CRP levels. In the patients with chronic SCI, somatosensory and visceral stimulation below the level of the lesion can lead to sympathetic overactivity and increased norepinephrine (Wang et al, 2007). Norepinephrine activates beta adrenoceptors which are expressed on immune cells causing the release of proinflammatory cytokines. Proinflammatory cytokines act in the liver to stimulate the production and release of acute phase proteins such as CRP (Nance et al., 2007; Lampert et al., 2008; Jänig, 2014). Many studies have found that good physical activity can reduce excessive sympathetic activity (Mueller et al., 2007). Excessive sympathetic activity at SCI C3-T6 levels can be reduced through physical activity, so CRP production will also decrease, as was found in this study where the active group had significantly lower CRP levels than the less active group.

Lymphoid organs such as the spleen and adrenal glands are innervated by sympathetic neurons originating from the entire region of the thoracolumbar spinal cord (T6-L1) (Cramer et al., 2005; Allison et al., 2015). This happens that patients with SCI levels C3-T6 experience a cut off of sympathetic signals to lymphoid organs, so that inflammation cannot be reduced. Physical exercise can reduce inflammatory markers by reducing cytokine release and also increasing antioxidant status (Hopps et al., 2011). Physical activity can reduce proinflammatory cytokines and then reduce CRP (Queiroz et al., 2020). At SCI levels C3-T6 where there was an increase in proinflammatory cytokines, the group that had a significantly active level of physical activity had better CRP levels than the less active group.

The higher the CRP level, the higher the risk of a person developing cardiovascular disease. The results of the study in table 3 show that the active group of SCI C3-T6 patients regardless of the condition where all four extremities are weak, have better hs-CRP levels than the less active group, so they have a lower risk of cardiovascular disease than the less active group. 


\subsubsection{C-reactive protein based on physical activity level on SCI level T7-below}

There was no significant difference in the T7-below group ( $p$ 0.165). CRP levels were not significantly different in patients with SCI level T7-below, both those with active and inactive physical activity levels. Cardiac sympathetic autonomic function is regulated at the T1-T6 level (Furlan et al., 2008). Patients with SCI level T7-below still have intact supraspinal sympathetic control of the heart. This causes SCI patients at this level to be able to control the excessive increase in sympathetic activity. SCI patients at T7-below also retain some sympathetic control of the adrenal glands and lymph nodes. Sympathetic autonomic activity for the spleen is regulated at the T6-T10 level and the adrenal glands are regulated at the T8-L1 level (Cramer et al., 2005). Inflammation that occurs can still be partially controlled by lymphoid organs which still receive some sympathetic innervation from the spinal cord. These factors explained that CRP levels were not significantly different in patients with SCI level T7-below, both those with active and less active levels of physical activity. The average hs-CRP value of chronic SCI patients in this study was above the normal value $(>1 \mathrm{mg} / \mathrm{L})$ both at high and low levels of injury.

Physical activity is not only determined by the type of mobilization but also other daily activities. This study compared hs-CRP levels based on the level of physical activity of chronic SCI patients using the IPAQ questionnaire assessment which included physical activity while sitting relaxed, physical activity in relation to mobilization, work, home/family care, sports and recreation. This study compared hs-CRP in each group of lesion levels based on the level of physical activity, so as to get an overview of the effect of physical activity level on hs-CRP in chronic SCI with high and low lesion levels. This comparison does not exist in previous journals. The drawback of this study is that the measurement of adipose tissue in this study did not use DXA, which can provide a more accurate picture of whole bodies fat. This study did not record the number of times the patient had an infection during a spinal cord injury and the last time the patient had an infection.

\section{Conclusion}

The levels of hs-CRP on the chronic SCI patients did not differ based on the level of injury in terms of age, body mass index, duration of injury, level of physical activity, etiology of SCI, AIS, and history of catheter use which were homogeneous in both groups. Chronic SCI patients with active physical activity levels had better hs-CRP than those who were less active in the SCI group of C3-T6.

\section{References}

Allison DJ, Ditor DS. 2015. Immune dysfunction and chronic inflammation following spinal cord injury. Spinal Cord advance online publication; doi:10.1038/sc.2014.184

Bauman WA, Raza M, Spungen AM, Machac J. 1994. Cardiac stress testing with thallium-201 imaging reveals silent ischemia in individuals with paraplegia.

Bravo G, Guizar-Sahagun G, Ibarra A, Centurion D, Villalon CM. Cardiovascular alterations after spinal cord injury: an overview. 2004. Curr Med Chem Cardiovasc Hematol Agents 2: 133-48.

Browning LM, Mugridge O, Dixon AK, Aitken SW, Prentice AM, Jebb SA. 2011. Measuring Abdominal Adipose Tissue: Comparison of Simpler Methods with MRI. Obes Facts 2011;4:9-15

Calvo-Infante RF, Narvaez-Rojas A, Padilla-Zambrano H, Hoz SS, Agrawal A, Moscote-Salazar LR. 2018. Cardiovascular complications associated with spinal cord injury. J Acute Dis 7(4): 139-144

Caspersen CJ, Powell KE, Christenson GM. 1985. Physical Activity, Exercise, and Physical Fitness: Definitions and Distinctions for Health-Related Research. Public Health Report Vol.100 No.2

Cramer GD, Darby SA. 2005. Neuroanatomy of autonomic nervous system in Basic and Clinical Anatomy of the Spine, Spinal Cord, and ANS $2^{\text {nd }}$ ed- E-Book. Elsevier mosby. Hal 459

El-Kotob R , Craven B C, Mathur S, Ditor D S, Paul Oh, Miyatani M, Verrier M C. 2018. Assessing Heart Rate Variability As a Surrogate Measure of Cardiac Autonomic Function in Chronic Traumatic 
Spinal Cord Injury. Top Spinal Cord Ibj Rehabil; 21(1):28-36.

Furlan JC, Fehlings MG. 2008. Cardiovascular complications after acute spinal cord injury: pathophysiology, diagnosis, and management. Neurosurg Focus 25(5).

Gibson AE, Buchholz AC, Martin Ginis KA, SHAPE-SCI Research Group. 2008. C-Reactive protein in adults with chronic spinal cord injury: increased chronic inflammation in tetraplegia vs paraplegia. Spinal Cord 46, 616-621

Goldstein RL, Walia P, Teylan M, Lazzari AA, Tun CG, Hart JE, Garshick E. 2017. Clinical factors associated with C-reactive protein in chronic spinal cord injury. Spinal Cord 55, 1088-1095

Hastuti, J. 2013. Anthropometry and Body Composition of Indonesian Adults: An Evaluation of Body Image, Eating Behaviours, and Physical Activity [Disertasi]. Queensland University of Technology.

Hopps E, Canino B, Caimi G. 2011. Effects of exercise on inflammation markers in type 2 diabetic subjects. Acta Diabetol (2011) 48:183-189

Huang CC, Liu CW, Weng MC, Chen TW, Huang MH. 2008. Association Of C-Reactive Prote In And Insulin Resistance In Patients With Chronic Spinal Cord Injury. J Rehabil Med 40: 819-822

Jänig W. 2014. Sympathetic nervous system and inflammation: A conceptual view. Auton.Neurosci-01623; page 11

Lampert R, Bremner JD, Su S, Miller A, Lee F, Cheema F, Goldberg J, Vaccarino V. 2008. Decreased heart rate variability is associated with higher levels of inflammation in middle-aged men. Am Heart $\mathrm{J}$ 156(4): 759.e1-759.e7.

Lee M, Myers J, Hayes A, Madan S, Froelicher VF, Perkash I, Kiratli BJ. 2005. C-Reactive Protein, Metabolic Syndrome, and Insulin Resistance in Individuals With Spinal Cord Injury. The Journal of Spinal Cord Medicine 28:1, 20-25

Majka DS, Chang RW, MD, Vu THT, Palmas W, Geffken DF, Liu K. 2009. Physical Activity and HighSensitivity C-Reactive Protein: The Multi-Ethnic Study of Atherosclerosis. Am J Prev Med 36(1): $56-62$.

Morse LR, Stolzmann K, Nguyen HP, Jain NB, Zayac C, Gagnon DR, Tun CG, MD, Garshick E. 2008. Association Between Mobility Mode and C-Reactive Protein Levels in Men With Chronic Spinal Cord Injury. Arch Phys Med Rehabil; 89(4): 726-731.

Mueller PJ. 2007. Exercise Training and Symphatetic Nervous System Activity: Evidence for Physical Activity Dependent Neural Plasticity. Clinical and Experimental Pharmacology and Physiology 34, 377-384

Myers J, Lee M, Kiratli J. 2007. Cardiovascular disease in spinal cord injury: an overview of prevalence, risk, evaluation, and management. Am J Phys Med Rehabil 86: 142-52.

Nance DM, Sanders VM. 2007. Autonomic Innervation and Regulation of the Immune System (1987-2007). Brain Behav Immun 21(6): 736-745.

Plaisance EP, Grandjean PW. 2006. Physical Activity and High-Sensitivity C-Reactive Protein. Sports Med 36 (5): 443-458

Queiroz CO, Pitanga F, Lotufo PA, Molina MDCB, Aquino EML, Almeida MCC. 2020. Amount of physical activity necessary for a normal level of high-sensitivity C-reactive protein in ELSA-Brasil: a crosssectional study. Sao Paulo Med J 138(1):19-26

Ruiz-Ramie JJ, Barber JL, Sarzynski MA. 2019. Effects of exercise on HDL functionality. Curr Opin Lipido 30:16-23.

Sproston NR and Ashworth JJ. 2018. Role of C-Reactive Protein at Sites of Inflammation and Infection. Front. Immunol. 9:754. doi: 10.3389/fimmu.2018.00754

Wang TD,Wang YH,Huang TS, Su TC, Pan SL, Chen SY. 2007. Circulating Levels of Markers of Inflammation and Endothelial Activation are Increased in Men with Chronic Spinal Cord Injury. J Formos Med Assoc Vol 106 No 11

Warburton DER, Eng JJ, Krassioukov A, Sproule S, the SCIRE Research Team. 2007. Cardiovascular health 
and exercise rehabilitation in spinal cord injury. Topics in Spinal Cord Injury Rehabilitation 13: 98122.

West CR, Mills P, Krassioukov AV. 2012. Influence of the neurological level of spinal cord injury on cardiovascular outcomes in humans: a meta-analysis. Spinal Cord 50(7):484-492.

Widhiyanto L, Martiana Ik, Primadenny AA, Donny P. 2018.Studi Epidemiologi Fraktur Vertebra Di Rsud Dr.Soetomo Surabaya Pada Tahun 2013-2017. Qanun Medika Vol. 3 No. 1

Vashist, SK, Venkatesh AG, Schneider EM, Beaudoin C, Luppa PB, Luong JHT. 2015. Bioanalytical advances in assays for C-reactive protein, Biotechnol Adv. http://dx.doi.org/10.1016/j.biotechadv.2015.12.010 